\title{
Intracranial Abscess Post Intravenous Misuse of Transdermal Fentanyl in an Immunocompetent Patient
}

\author{
Hazel Serrao-Brown \\ The Canberra Hospital, Canberra, Australia \\ Email: Hazel.G.Serrao-Brown@act.gov.au
}

How to cite this paper: Serrao-Brown, H. (2018) Intracranial Abscess Post Intravenous Misuse of Transdermal Fentanyl in an Immunocompetent Patient. Open Journal of Modern Neurosurgery, 8, 143-146. https://doi.org/10.4236/ojmn.2018.82012

Received: February 10, 2018

Accepted: March 5, 2018

Published: March 8, 2018

Copyright $\odot 2018$ by author and Scientific Research Publishing Inc. This work is licensed under the Creative Commons Attribution International License (CC BY 4.0).

http://creativecommons.org/licenses/by/4.0/

\begin{abstract}
Infections, including intracranial abscesses, are a well-known and potentially serious complication of intravenous drug abuse. There have been increasing reports of intravenous misuse of transdermal Fentanyl, with severe sequelae. Intracranial abscesses in intravenous drug users tend to involve uncommon pathogens, and may present with atypical symptoms, which can lead to delayed diagnosis and inadequate treatment. We present the case of a 28-year-old female, with a history of intravenous misuse of transdermal Fentanyl, who was found to have intracranial abscesses with Fusobacterium nucleatum and Nocardia species, causing significant vasogenic oedema and mass effect.
\end{abstract}

\section{Keywords}

Intracranial Abscess, Transdermal Fentanyl, Intravenous Drug Use

\section{Introduction}

Infections are a well-known and potentially serious complication of intravenous drug abuse [1]. These infections include intracranial abscesses, which may occur due to the use of non-sterile injections and contamination of the injected substance, as well as life-style practices and impaired host immunity [1]. Most reported cases of drug abuse related intracranial abscesses are due to injection of illegal drugs, such as heroin and amphetamines. There have been increasing reports of intravenous abuse of transdermal Fentanyl, with the main complication being related to respiratory depression, with no published reports of intracranial abscesses related to this [2] [3]. Moreover, most intracranial abscesses involve Staphylococcus aureus, Group A streptococci and fungal species [1]. Nocardia and Fusobacterium species have been rarely implicated in intracranial abscesses, 
as they are more commonly found in pulmonary, periodontal and some intra-abdominal infections, and tend not to occur in immunocompetent patients [4] [5]. We present the case of a 28-year-old female with atypical intracranial abscesses and a history of intravenous use of transdermal Fentanyl patches.

\section{Case Description}

A 28-year-old female, with a history of intravenous drug use, presented to the emergency department with a two day history of worsening headaches and photophobia. She denied any fevers, nausea or rash. She denied a history of migraines, but did report recurrent intravenous use of transdermal Fentanyl patches. On physical examination, she was afebrile and haemodynamically stable, with an initial Glasgow Coma Score (GCS) of 14. Neurological examination was unremarkable, with pupils equal and reactive and no focal neurological deficits. Laboratory testing demonstrated raised white cell count, neutrophilia and raised inflammatory markers. She was initially thought to have a migraine and was managed accordingly. However, within a few hours, she reported worsening of her headache and persistent photophobia. She also began to have mildly slurred speech. Contrast CT brain demonstrated two large peripherally enhancing lesions within the right frontal lobe, with associated mass effect and obstructive hydrocephalus (Figure 1).

Neurosurgical review was obtained, and the patient was taken to theatre for urgent intervention. A right frontal craniotomy was performed. The brain was found to be globally oedematous, with extensive purulent fluid within the Sylvian fissure. Two thick walled abscesses were found within the frontal lobe, copious amounts of purulent fluid were washed out, with a specimen sent for microbiological workup. The wound was closed.

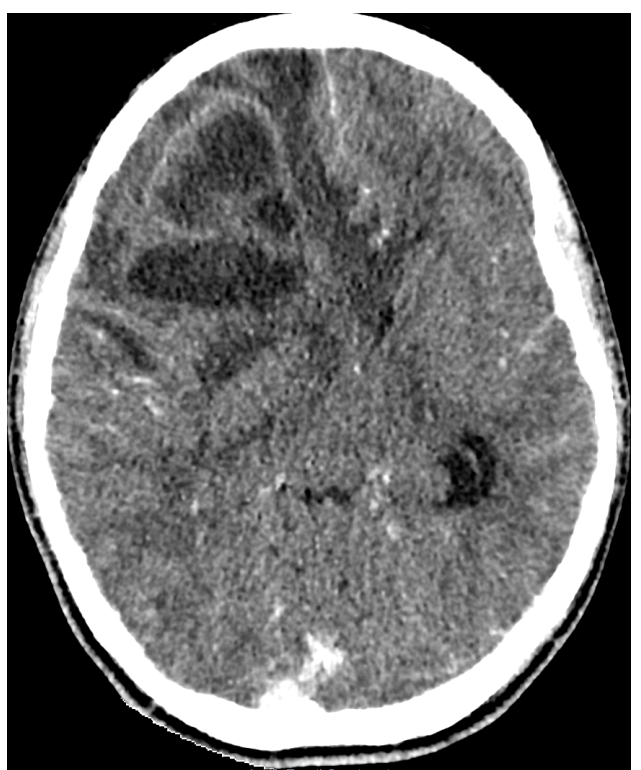

Figure 1. Contrast CT scan of the brain at presentation, demonstrating two frontal lobe abscesses and mass effect. 
Streptococcus milleri group, Nocardia and Fusobacterium nucleatum groups were cultured. The patient was treated with intravenous Trimethoprim-Sulfamethoxazole, Meropenem, Ceftriaxone and Vancomycin, as well as Dexamethasone. She was extubated 3 days later without event. Post-operative imaging revealed decompression of the frontal lobe abscess, with ongoing vasogenic oedema. Clinically, this patient did not have any focal neurological deficits post-operatively. Further work-up did not reveal any other potential infectious sources, such as sinusitis, valvular vegetations, otogenic or odontogenic infections.

\section{Discussion}

Infectious complications of substance abuse are a frequent cause of hospital admissions, with atypical poly-microbial pathogens, and uncommon types and sites of infection [1]. It is important to be aware of this while choosing appropriate antimicrobial therapies, as delay in effective treatment may worsen patient outcomes [4]. Central nervous system infections and related complications remain a major source of mortality and morbidity, and necessitate a multidisciplinary treatment approach [5] [6].

Frontal lobe abscesses frequently present with subtle clinical symptoms, leading to this lobe being referred to as the "silent area" of the brain [5]. Neuro-imaging, such as a contrast CT scan, is essential for the diagnosis of intracranial abscesses, and is useful to aid early characterisation, localisation and staging of these abscesses [5]. Surgical intervention is required not only to reduce mass effect, but also to facilitate confirmation of the diagnosis, characterisation of the involved pathogens, and to guide appropriate antibiotic therapy [5]. Infectious diseases input and microbiological studies are essential in guiding antibiotic choice and duration [5]. This patient, with two large frontal abscesses and associated mass effect, required prompt diagnosis, urgent neurosurgical decompression and appropriate antibiotic cover. Experimental studies have demonstrated the effect of Dexamethasone in reducing cerebral oedema associated with intracranial abscesses, although it may impair the host's immune response as well as reduce antibiotic efficacy [7].

Nocardial intracranial abscesses are rare, however studies have shown a mortality rate of greater than $47 \%$ in patients with these abscesses [8] [9]. Fusobacterium species are found in around $6 \%$ of intracranial abscess, and most patients tend to survive [4] [10]. In this case, given no detection of an extracranial focus of infection, it is possible that parenteral inoculation had occurred.

Fentanyl misuse has become increasingly common in recent years and continues to rise [3]. This is an interesting case highlighting a potentially severe complication of intravenous use of transdermal Fentanyl. Clinicians should be aware of this potential diagnosis, and should attempt to rule out intracranial abscess in intravenous drug users who present with significant neurological symptoms, even if these are atypical, and arrange urgent investigation and intervention to prevent impending sequelae. 


\section{Conclusion}

The potential for the abuse of Fentanyl has been well established. This report could serve to raise awareness of the possibility of the serious complication of developing an intracranial abscess secondary to injecting transdermal Fentanyl, as well as the rare pathogens involved. Severe cases require a multidisciplinary approach with urgent intervention as well as early microbiological studies and tailoring of antibiotic treatment.

\section{References}

[1] Kaushik, K.S., Kapila, K. and Praharaj, A.K. (2011) Shooting up: The Interface of Microbial Infections and Drug Abuse. Journal of Medical Microbiology, 60, 408-422. https://doi.org/10.1099/jmm.0.027540-0

[2] Kuhlman Jr., J.J., McCaulley, R., Valouch, T.J. and Behonick, G.S. (2003) Fentanyl Use, Misuse, and Abuse: A Summary of 23 Post-Mortem Cases. Journal of Analytical Toxicology, 27, 499-504. https://doi.org/10.1093/jat/27.7.499

[3] Schauer, C.K., Shand, J.A. and Reynolds, T.M. (2015) The Fentanyl Patch Boil-Up-A Novel Method of Opioid Abuse. Basic and Clinical Pharmacology and Toxicology, 117, 358-359. https://doi.org/10.1111/bcpt.12412

[4] Hischebeth, G.T., Keil, V.C., Gentil, K., et al. (2014) Rapid Brain Death Caused by a Cerebellar Abscess with Fusobacterium nucleatum in a Young Man with Drug Abuse: A Case Report. BMC Research Notes, 7, 353. https://doi.org/10.1186/1756-0500-7-353

[5] Miranda, H.A., Castellar-Leones, S.M., Elzain, M.A. and Moscote-Salazar, L.R. (2013) Brain Abscess: Current Management. Journal of Neurosciences in Rural Practice, 4, S67-81. https://doi.org/10.4103/0976-3147.116472

[6] Muzumdar, D. (2011) Central Nervous System Infections and the Neurosurgeon: A Perspective. International Journal of Surgery, 9, 113-116. https://doi.org/10.1016/j.ijsu.2010.11.001

[7] Yildizhan, A., Pasaoglu, A. and Kandemir, B. (1989) Effect of Dexamethasone on Various Stages of Experimental Brain Abscess. Acta Neurochirurgica, 96, 141-148. https://doi.org/10.1007/BF01456174

[8] Geiseler, P.J. and Anderson, B.R. (1979) Results of Therapy in Systemic Nocardiosis. The American Journal of the Medical Sciences, 278, 188-194. https://doi.org/10.1097/00000441-197911000-00001

[9] Duran, E., Lopez, L., Martinez, A., et al. (2001) Primary Brain Abscess with Nocardia otitidiscaviarum in an Intravenous Drug Abuser. Journal of Medical Microbiology, 50, 101-103.

[10] Chakvetadze, C., Purcarea, A., Pitsch, A., et al. (2017) Detection of Fusobacterium nucleatum in Culture-Negative Brain Abscess by Broad-Spectrum Bacterial $16 \mathrm{~S}$ rRNA Gene PCR. IDCases, 8, 94-95. https://doi.org/10.1016/j.idcr.2017.04.013 